\title{
HERRAMIENTA NUMÉRICA PARA LA EVALUACIÓN DE DESPLAZAMIENTOS DE DOS FASES EN MEDIOS POROSOS USANDO TOMOGRAFÍA COMPUTARIZADA
}

\author{
Crisóstomo Barajas-Solano ${ }^{1^{*}}$; Samuel Muñoz ${ }^{2}$; Nicolás Santos ${ }^{2}$; Henry Arguello ${ }^{1}$
}

DOI: http://dx.doi.org/10.18273/revbol.v39n3-2017007 @) (1)

Forma de citar: Barajas-Solano, C., Muñoz, S., Santos, N., y Arguello, H. 2017. Herramienta numérica para la evaluación de desplazamientos de dos fases en medios porosos usando tomografía computarizada. Boletín de Geología, 39(3): 99-109.

\section{RESUMEN}

La producción anual de crudo en Colombia ha aumentado año tras año, hasta alcanzar los 367 millones de barriles por año y una reserva probada de 2002 millones de barriles, en el 2016. Esto se debe a la intensificación de actividades de explotación de yacimientos maduros y de crudo pesado, y el uso de Técnicas de Recobro Mejorado (EOR). Estas buscan reducir la saturación de crudo en un yacimiento mediante la inyección de fluidos como solventes, polímeros, vapor y aire, principalmente. El porcentaje de recuperación en Colombia alcanzado con técnicas EOR es cercano al $21 \%$. No obstante, su efectividad depende de: las características del crudo presente en el yacimiento, el mecanismo de empuje predominante en este, presión y temperatura, además de las propiedades petrofísicas de la roca que forma el yacimiento. Este trabajo aborda la estimación de las propiedades petrofísicas dinámicas de muestras de roca del horizonte productor mediante el uso de Tomografía Computarizada (acorde a sus siglas en inglés CT). Para esto se propone un conjunto de cinco algoritmos numéricos para la estimación de la porosidad efectiva, saturación, frente de avance, volumen atrapado y factor de recobro durante el desplazamiento de dos líquidos, crudo y agua, en el interior del medio poroso. El objetivo de la caracterización dinámica, mediante CT, es comprender y analizar los parámetros que influyen en el éxito de un desplazamiento de crudo en un medio poroso como parte de un esquema de producción mejorado en yacimientos maduros y de crudo pesado.

Palabras clave: tomografía computarizada de rocas; desplazamiento de dos fases; recobro mejorado; algoritmos numéricos.

\section{NUMERICAL TOOL FOR THE EVALUATION OF TWO PHASE DISPLACEMENTS IN POROUS MEDIA USING COMPUTERIZED TOMOGRAPHY}

\begin{abstract}
The annual production of crude oil in Colombia has increased, from year to year, to 367 million barrels per year and a proven reserve of 2002 million barrels in 2016. This is due to the intensified production of mature, and heavy crude, oil fields and the use of Enhanced Oil Recovery Techniques (EOR). These seek to reduce the saturation of crude oil in a reservoir by injecting fluids such as solvents, polymers, steam and air, mainly. The percentage of recovery in Colombia achieved with EOR techniques is close to $21 \%$. However, its effectiveness depends on: the characteristics of the oil present in the reservoir, the thrust mechanism prevailing in this, pressure, and temperature, in addition to the petrophysical properties of the rock that forms the reservoir. This work deals with the estimation of the dynamic petrophysical properties of rock samples from the producer horizon using Computed Tomography (CT). For this, a set of five numerical algorithms is proposed for the estimation of the effective porosity, saturation, forward front, trapped volume, and recovery factor during the displacement of two liquids, crude and water, inside the porous medium. The purpose of the dynamic characterization using CT is to understand and analyze the parameters that influence the success of crude oil displacement in a porous medium as part of an improved production scheme in mature, and heavy crude, oil fields.
\end{abstract}

Keywords: Computerized tomography of rocks; two-phase displacement; improved recovery; numerical algorithms.

\footnotetext{
${ }^{1}$ Escuela de Ingeniería de Sistemas e Informática, Universidad Industrial de Santander, Bucaramanga, Santander, Colombia. (*)crisostomo.barajas@correo.uis.edu.co; henarfu@uis.edu.co

2 Escuela de Ingeniería de Petróleos, Universidad Industrial de Santander, Bucaramanga, Santander, Colombia. samuel@uis.edu.co; nicolas@uis.edu.co
} 


\section{INTRODUCCIÓN}

Las reservas mundiales de petróleo han disminuido sus niveles en la última década (OPEC, 2016). En Owen et al. (2010) se calculó que entre los años 2000 y 2010 las reservas mundiales de crudos convencionales decrecieron en 250.000 millones de barriles (22\%). Colombia, por el contrario, aumentó su producción a 367 millones de barriles por año en el 2016, gracias a la mayor explotación de yacimientos maduros y de crudo pesado, según la Agencia Nacional de Hidrocarburos (ANH, 2016).

El crudo pesado se caracteriza por ser más denso y viscoso, por lo tanto con mayor resistencia a fluir y con mayor concentración de sulfuros y otros contaminantes que el crudo convencional. Su refinación requiere de una mayor cantidad de energía y genera mayores problemas en su extracción y transporte (Speight, 1997). Sin embargo, se estima que las reservas mundiales de crudo pesado duplican las reservas de crudos convencionales (Meyer et al., 2007).

Las técnicas de recobro mejorado permiten incrementar la producción de un yacimiento maduro o de crudo pesado al inyectar aire, gases, solventes o polímeros, principalmente al interior del yacimiento. Colombia es un país relativamente nuevo en la aplicación de esta clase de procesos y tecnologías. Países como Venezuela y Canadá son líderes en explotación de este tipo de yacimientos. Sin embargo, las condiciones geológicas en estos países, y las características de los hidrocarburos allí extraídos, son diferentes a las encontradas en Colombia (Wheeler et al., 1996; SGC, 2015; Hackley et al., 2006), por lo que la transferencia tecnológica no ha sido inmediata.

La eficiencia de las técnicas de recobro mejorado aumenta en la medida que se conocen las propiedades petrofísicas del yacimiento. Actualmente la caracterización petrofísica de muestras de roca se realiza según el estándar API RP-40, el cual incluye una serie de técnicas clásicas, en su mayoría destructivas y/o invasivas, que no permiten observar el desplazamiento de agua o crudo al interior de esta, solo inferirlo.

La Tomografía Computarizada (acorde a sus siglas en inglés CT) de rocas, en contraste a la norma API RP40 , es una técnica que permite observar la estructura interna de una muestra de roca, al interpretar las variaciones en las atenuaciones de un haz de rayos-X que atraviesa la muestra. Estas atenuaciones son provocadas por variaciones en la densidad del material estudiado, lo cual permite analizar el desplazamiento de fluidos y fenómenos asociados al desplazamiento de crudo debido a agentes de recobro mejorado.

La cuantificación de las propiedades petrofísicas dinámicas busca analizar, a escala de laboratorio, la evolución del desplazamiento de crudo al interior del medio poroso. La estimación de la porosidad efectiva permite evaluar el potencial de desplazamiento de dos fases líquidas en el interior del medio poroso; la variación de la saturación en el tiempo permite estimar la cantidad de crudo desplazado por unidad de tiempo, y por tanto, evaluar el desempeño de la técnica EOR. Estudiar el desplazamiento crudo al interior de una muestra de roca, usando imágenes CT, permite incrementar el factor de recobro más allá del $21 \%$, promedio actual para los pozos que se operan actualmente en el país (Castro et al., 2010).

El presente documento se compone de las siguientes secciones: se presenta una introducción a los principios físicos de la Tomografía Computarizada, y su aplicación en muestras de rocas. A continuación, se presentan las muestras de roca usadas en el desarrollo de los algoritmos numéricos propuestos para el procesamiento de las imágenes CT y, por último, los resultados y conclusiones obtenidas.

\section{Tomografía Computarizada de Rocas}

La Tomografía Computarizada es un método no invasivo que permite observar la estructura interna de un objeto, en una sección transversal específica. Esta técnica requiere del escaneo del objeto en diferentes puntos, generando imágenes bidimensionales, con las que se pueden realizar reconstrucciones volumétricas (Buzug, 2008). Sus aplicaciones incluyen la detección de sustancias peligrosas y explosivos (Cozzini et al., 2012; Strecker, 1998), control de calidad en la industria de manufactura (Smith, 1999), detección de drogas ilegales (Pani et al., 2009), imágenes diagnosticas médicas y antropología (Blau et al., 2008; Borkan et al., 1983; Fajardo y Müller, 2001), ciencias forenses (Kellinghaus et al., 2010; Thali et al., 2003a; Thali et al., 2003b), arqueología (Uda et al., 2005), paleontología (Tafforeau et al., 2006), entre otras. La adquisición de imágenes por tomografía tiene una ventaja sobre la resonancia magnética, dado que esta última requiere que el objeto escaneado contenga iones de hidrogeno en forma de agua. Esto limita la aplicación de la resonancia magnética en estudios de geología, antropología y arqueología (Buzug, 2008). 
Un sistema tradicional de adquisición de medidas de CT consta de una fuente de rayos-X y una línea de detectores que giran alrededor del objeto de estudio, siguiendo una trayectoria circular sobre el eje axial del objeto escaneado. El haz de rayos-X atraviesa entonces el objeto escaneado de manera transversal. Dependiendo de la trayectoria particular que toma cada rayo, este será atenuado en mayor o menor grado por el objeto. La absorción local de energía se mide con el conjunto de detectores. Como las proyecciones que se obtienen en una sola dirección no son suficientes para determinar la distribución espacial del objeto (especialmente si se trata de un objeto tridimensional) se hace necesario muestrear el objeto desde diferentes ángulos. Para modelar las proyecciones que se generan con la estructura de los rayos-X se hace uso de la transformada Radon, introducida por Johann Radon en 1917, modela el proceso de transmisión de un rayo a través del objeto con integrales de línea (Buzug, 2008). De manera general, para un ángulo de proyección y una posición de la fuente, se da la transformada Radon como (Buzug, 2008):

$$
p(s)=\int_{0}^{s} \mu(\eta) d \eta,
$$

Donde $\mu(\eta)$ representa los coeficientes de atenuación de las diferentes densidades del objeto, $s$ la longitud de integración, y $p(s)$ la proyección de la energía del haz de rayos X sobre la energía captada por los sensores. La FIGURA 1 muestra un ejemplo de discretización de los coeficientes de atenuación, de manera que la ecuación 1 se puede reescribir como:

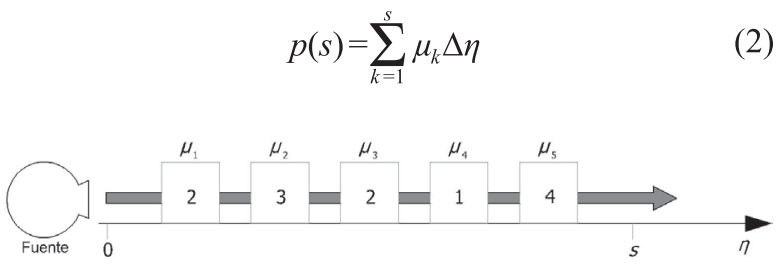

FIGURA 1. Ley de Lambert-Beer: Atenuación de la intensidad de radiación al pasar a través de un objeto no homogéneo (Modificado de Buzug, 2008).

Si la fuente y los detectores se mueven con el mismo radio desde el objeto, la longitud de la integración $s$ es constante. De manera más general, bajo cualquier ángulo $\gamma$ de proyección se define un sistema de rotación $(\varepsilon, \eta)$ como:

$$
p_{\gamma}(s)=\int_{0}^{s} \mu(\varepsilon, \eta) d \eta
$$

Cuando se captan todas las mediciones se pueden disponer en un vector $\mathbf{p}$, el cual contiene todos los valores adquiridos por los detectores, y está dado por:

$$
\begin{gathered}
\mathbf{p}=\mathbf{A f} \\
\mathbf{p}_{\gamma}(\varepsilon)=\mathbf{R}\{\mathbf{f}(x, y)\}
\end{gathered}
$$

Donde $\boldsymbol{A}$ es la matriz que modela el sistema (transformación al espacio Radon, $\boldsymbol{R}$ ) y $\boldsymbol{f}$ contiene todos los valores de grises de la rejilla (imagen en el espacio cartesiano $(\mathrm{x}, \mathrm{y})$ ) que modela el objeto de estudio. Una vez se tienen las mediciones, se aplica la transformada inversa de Radon para obtener la imagen reconstruida (Buzug, 2008). La imagen resultante es la representación de la estructura interna de la muestra escaneada en un único corte transversal. La muestra a escanear se debe entonces desplazar en su eje axial para lograr varias imágenes transversales de principio a fin, obteniendo un cubo de datos de $l$-cortes transversales de resolución espacial $m \times n$, donde $l, m$ y $n$ dependen de la resolución del tomógrafo usado. Este proceso de muestreo está limitado por la tasa de muestreo de Nyquist, según la cual la resolución está determinada por el sensor usado en el proceso de muestreo (Shannon, 1949).

El principal campo de aplicación de la Tomografía Computarizada ha sido el diagnóstico médico, aunque también ha sido usada extensivamente en la industria de hidrocarburos para el estudio de muestras de roca de yacimientos de petróleo por cerca de 20 años (Bansal e Islam, 1991; Hunt et al., 1998; Kantzas, 1990; Wellington y Vinegar, 1987; Withjack, 1988). Las aplicaciones de la Tomografía Computarizada en la industria de hidrocarburos se concentran principalmente en el escaneo de medios porosos sin comprometer su integridad física ni humectabilidad. La evaluación cualitativa de las imágenes CT de muestras de roca provee información de la heterogeneidad, cambios litológicos, fracturas, etc., mientras que la evaluación cuantitativa permite cuantificar la densidad aparente y porosidad, entre otras propiedades petrofísicas (Shameem y Khamees, 2004).

La visualización de flujo de fluidos constituye uno de los usos más predominante de la tecnología CT en la industria de hidrocarburos. Esta requiere el uso de trazadores radiopacos (dopantes) para monitorear el movimiento de fluidos dentro de una muestra de roca. Los dopantes proveen suficiente información del contraste entre las diferentes fases líquidas al interior de la muestra de roca, y permiten cuantificar la distribución de saturación de fluidos al interior de la misma (Withjack, 1988). 


\section{MATERIALES Y MÉTODOS}

\section{Muestras de roca de yacimientos maduros y de crudo pesado}

Para el presente trabajo de investigación se tomaron 3 muestras cilíndricas de roca, de 5" de largo por 1,5" de diámetro. Estas muestras de roca fueron obtenidas del pozo C-16, localizado en el departamento del Magdalena, Colombia. La densidad y composición para cada muestra de roca fueron obtenidas usando técnicas estándar reportadas en la norma API RP-40, tal y como se reporta en la TABLA 1.

TABLA 1. Muestras de roca tomadas del pozo C-16, Pozo Colorado, Magdalena (Colombia).

\begin{tabular}{|c|c|c|c|c|}
\hline Muestra & $\begin{array}{l}\text { Profundidad } \\
\text { (pies) }\end{array}$ & $\begin{array}{l}\text { Densidad } \\
(\mathrm{g} / \mathrm{cc})\end{array}$ & $\begin{array}{l}\text { Composición } \\
\text { mineralógica }\end{array}$ & Porosidad \\
\hline B2-1 & 3708 & $1,6-1,80$ & $\begin{array}{c}\text { Cuarzo } \\
\text { predominante }\end{array}$ & $19-21 \%$ \\
\hline H-B1 & 3856 & 2,00 & $\begin{array}{c}\text { Cuarzo } \\
\text { predominante }\end{array}$ & $19-21 \%$ \\
\hline 136 & 3956 & 2,10 & $\begin{array}{c}\text { Cuarzo } \\
\text { predominante }\end{array}$ & $19-21 \%$ \\
\hline
\end{tabular}

La Tomografía Computarizada permite estimar la saturación de agua y crudo al interior de una muestra de roca. Esto es, conocer la cantidad del espacio poroso ocupado por cada fase. Eso se logra midiendo la variación en los números $\mathrm{CT}$ entre dos instantes diferentes de tiempo. A una mayor diferencia entre los números CT del agua y crudo aumenta la resolución de la estimación numérica. Para lograr este contraste se usa agua dopada con yodo, cuyo número $\mathrm{CT}$ promedio es de $2241 \mathrm{HU}$, mientras que el del crudo es de $236 \mathrm{HU}$. Por último, es necesario conocer el valor de atenuación de la roca en saturación máxima (para ambas fases) como valor de referencia.

El experimento de caracterización dinámica parte de una muestra de roca seca $\left(S_{0}\right)$, a la cual se le inyecta crudo a tasa constante, hasta saturarla por completo $\left(S_{0=1}\right)$. En este estado se realiza el primer escaneo CT $\left(C T_{o=1}^{R}\right)$. Posteriormente, la muestra de roca es restaurada a su estado inicial $\left(S_{0}\right)$, y se procede a inyectar agua dopada, a una tasa constante, hasta saturarla por completo $\left(S_{w=1}\right)$. De nuevo se realiza un escaneo completo de este nuevo estado $\left(C T_{w=1}^{R}\right)$. El objetivo es obtener los dos estados de referencia de máxima saturación. Esto es, la máxima fracción del espacio poroso que puede ser ocupada por cada uno de las fases líquidas.

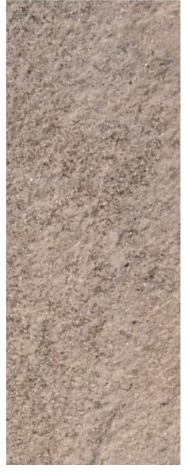

A

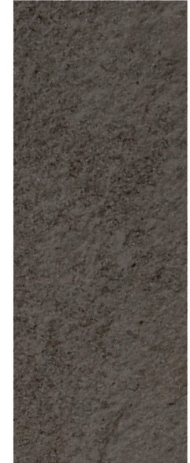

B

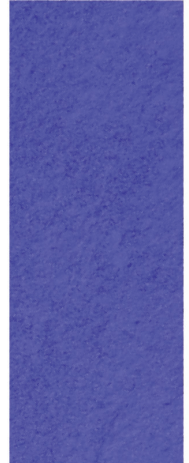

C

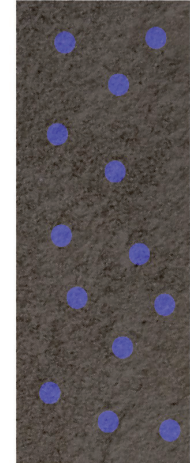

D
FIGURA 2. Preparación de una muestra de roca a condiciones de yacimiento: A. Muestra de roca seca $\left(S_{0}\right)$. B. Saturada completamente de crudo $\left(S_{\mathrm{o}=1}\right)$. C. Restaurada y saturada completamente de agua dopada $\left(S_{w=1}\right)$. D. saturada de crudo y agua irreducible $\left(S_{\text {wirr }}\right)$.

A continuación, se empieza a inyectar crudo, a tasa constante, hasta desplazar el mayor volumen posible de agua de la roca. Este estado se conoce como saturación de agua irreducible $\left(S_{\text {wirr }}\right)$, donde no es posible retirar más agua del interior de la roca debido a que esta ha quedado atrapada dentro de los poros de la roca. El estado $S_{\text {wirr }}$ simula las condiciones de la roca en el yacimiento, previo a su extracción, y sirve como referencia para estimar la evolución de la saturación de crudo al interior de la roca durante el experimento de desplazamiento. La FIGURA 2 representa los diferentes estados de la muestra de roca durante su preparación para la prueba de desplazamiento de dos fases.

Con la roca en estado $S_{\text {wirr }}$ se procede a inyectar agua dopada, a tasa constante, hasta lograr desplazar el mayor volumen posible de crudo del interior de la roca. Este estado se conoce como saturación de crudo irreducible $\left(S_{\text {orr }}\right)$, y simula un yacimiento después de la extracción de crudo al usar técnicas de recobro mejorado. Durante el desplazamiento del crudo se realiza una serie de muestreos CT, generando un conjunto de cubos de datos temporales en cada muestreo. El factor de recobro se estima entonces como la relación entre la concentración de crudo entre los estados $S_{\text {wirr }}$ y $S_{\text {orr }}$.

Cada cubo de datos, generado en cada escaneo CT, genera una serie de imágenes transversales (slides o cortes transversales) de la muestra de roca. Para este trabajo se tomaron cortes con una resolución espacial de $155 \mu \mathrm{m} \times 155 \mu \mathrm{m}$ y un distanciamiento de $340 \mu \mathrm{m}$ entre cortes. Es necesario considerar que el tamaño de los poros es mucho menor que la resolución espacial de los cortes. Se considera entonces que el espacio poroso $(\phi)$, en cada voxel de $155 \mu \mathrm{m} \times 155 \mu \mathrm{m} \times 340 \mu \mathrm{m}$ es la suma de todos los poros presentes en ese voxel. 
La segunda consideración a tener en cuenta es la caracterización del crudo usado y su comportamiento al desplazarse al interior de la roca escaneada. Crudos con diferentes propiedades (viscosidad, grados API, etc.) lograrán diferentes grados de saturación y se desplazarán a diferentes velocidades al interior de la roca. Al comparar el comportamiento de diferentes tipos de crudo, al desplazarse por diferentes tipos de roca, se puede extrapolar información importante del comportamiento de un yacimiento de crudo, y la consiguiente eficiencia de la extracción de crudo, sin la necesidad de incluir de manera explícita la caracterización del crudo dentro del modelo numérico.

El experimento de desplazamiento presentado en este trabajo fue propuesto por Kantzas (1990), al igual que las relaciones entre valor CT, saturación, densidad y cantidad másica. Los algoritmos presentados a continuación parten de los lineamientos de Kantzas (1990) y amplían su uso a grandes colecciones de cubos de datos. Sin embargo, algoritmos como la estimación de la posición del frente de avance, o la forma como se trata y analiza la información son las principales innovaciones que intenta aportar el presente trabajo.

\section{Algoritmo de estimación de porosidad efectiva}

El primer algoritmo es la estimación numérica de la porosidad efectiva de la muestra de roca ante la presencia del agua dopada y el crudo. Para esto se usan las imágenes CT de la roca totalmente saturada de crudo $\left(S_{\mathrm{o}=1}\right)$ y totalmente saturada de agua $\left(S_{\mathrm{w}=1}\right)$.

La idea principal es que el espacio disponible (espacio poroso, $\phi$ ), que antes estaba ocupado por aire, sea ocupado ahora por agua o crudo. Se puede definir entonces la porosidad, $\phi$, como la relación entre los dos estados de saturación de la roca, considerando la diferencia entre los valores $\mathrm{CT}$ de ambos fluidos, $C T_{o}$ y $C T_{w}$, así:

$$
\phi_{i}=\frac{C T_{o i}^{R}-C T_{w i}^{R}}{C T_{o}-C T_{w}}
$$

donde $C T_{o i}^{R}$ y $C T_{w i}^{R}$ son los valores $\mathrm{CT}$ promedio, para cada i-corte transversal, de la muestra de roca saturadas de crudo y agua dopada respectivamente; $\phi_{i}$ es la porosidad promedio de la roca, para ese corte. De nuevo, se promedian los valores CT de la muestra de roca por corte para evitar errores numéricos y de aproximación. El procedimiento para la estimación de la porosidad de una muestra de roca, usando CT, se presenta en el ALGORITMO 1.
ALGORITMO 1. Estimación de porosidad

Entrada: Cubos de datos CT de una muestra de roca totalmente saturada de crudo, $C T_{o=1}^{R}$, y totalmente saturada de agua, $C T_{w=1}^{R}$, a alta energía. Valores CT del crudo, $C T_{o}$, y del agua dopada, $C T_{w}$.

Salida: Porosidad de la roca, $\phi_{i}$, por cada corte transversal.

1. para cada i-corte transversal del cubo de datos hacer

2. promediar el valor de los números $\mathrm{CT}$ del i-corte de la muestra de roca saturada de crudo, $C T_{o=1}^{R}$

3. promediar el valor de los números $\mathrm{CT}_{R}$ del i-corte de la muestra de roca saturada de agua, $C T_{w=1}^{R}$

4. resolver

$$
\phi_{i}=\frac{C T_{o i}^{R}-C T_{w i}^{R}}{C T_{o}-C T_{w}}
$$

5. fin para

\section{Algoritmo de estimación de saturación}

Es posible conocer el estado de saturación de crudo y agua en el interior de la roca, en cualquier momento. Para esto se requiere tener como referencia la roca saturada completamente de crudo $\left(C T_{o}^{R}\right)$ y agua dopada $\left(C T_{w}^{R}\right)$, ademas de los valores $C \mathrm{~T}$ del crudo $\left(C T_{o}\right)$ y agua dopada $\left(C T_{w}\right)$.

La saturación de crudo al interior de la roca, en cada i-corte transversal y j-instante de tiempo, se determina así:

$$
S_{o i j}^{R}=\frac{C T_{i j}^{R}-C T_{i w}^{R}}{C T_{o}-C T_{w}},
$$

donde $C T_{i j}^{R}$ es el promedio de los valores CT de cada i-corte transversal de la roca durante el desplazamiento de dos fases, para cada j-instante de tiempo; y $C T_{i w}^{R}$ es el promedio de los valores $\mathrm{CT}$ de cada i-corte transversal de la roca saturada completamente de agua. Esto es, el espacio poroso que se encontraba ocupado por agua dopada ahora es ocupado crudo, y viceversa.

La saturación de agua al interior de la roca, en cada i-corte transversal y j-instante de tiempo, se determina así:

$$
S_{w i j}^{R}=\frac{C T_{i j}^{R}-C T_{i o}^{R}}{C T_{w}-C T_{o}},
$$

donde $C T_{i o}^{R}$ es el promedio de los valores CT de cada i-corte transversal de la roca saturada completamente de crudo. El procedimiento para la estimación de las saturaciones de agua dopada y crudo, al interior de la muestra de roca, se presenta en el ALGORITMO 2. 
ALGORITMO 2. Estimación de saturación de crudo y agua dopada en la muestra de roca para cualquier corte transversal e instante temporal

Entrada: Cubos de datos CT de una muestra de roca totalmente saturada de crudo, $C T_{o}^{R}$, y totalmente saturada de agua, $C T_{w}^{R}$, a alta energía. Valores CT del crudo, $C T_{o}$, y del agua dopada, $C T_{w}$. Conjunto de cubos de datos CT de la muestra de roca durante el desplazamiento de dos fases, tomados a diferentes instantes temporales, $C T^{R}$.

Salida: Concentración de crudo, $S_{o i j}^{R}$, y agua, $S_{w i j}^{R}$ en la roca para cada i-corte transversal y j-instante de tiempo.

1. para cada $j$-instante temporal hacer

2. para cada $\mathrm{i}$-corte transversal hacer

3. promediar el valor de los números $\mathrm{CT}$ de la muestra de roca en el i-corte transversal, $C T_{i j}^{R}$

4. resolver

\section{5. fin para}

$$
\begin{aligned}
S_{o i j}^{R} & =\frac{C T_{i j}^{R}-C T_{i w}^{R}}{C T_{o}-C T_{w}}, \\
S_{w i j}^{R} & =\frac{C T_{i j}^{R}-C T_{i o}^{R}}{C T_{w}-C T_{o}},
\end{aligned}
$$

6. fin para

\section{Algoritmo de estimación del frente de avance}

El resultado del ALGORITMO 2 es una superficie de saturación de agua dopada, o crudo, al interior del medio poroso vs tiempo vs corte transversal. A partir de esta curva se puede estimar la posición del frente de avance como la mayor variación de la saturación de agua dopada, o crudo, para cada instante temporal. Es indiferente usar la superficie de saturación de crudo o agua dopada en el cálculo del frente de avance dado que se cumple la siguiente relación lineal:

$$
S_{o i j}^{R}+S_{w i j}^{R}=1
$$

Por lo tanto, se tiene que la posición del frente de avance, para cada j-instante de tiempo, se puede estimar como el mayor valor absoluto de la derivada temporal de la saturación de crudo, así:

$$
F A_{j}=\max \left(\mid \frac{d S_{o i j}^{R}}{d t}\right), \forall i
$$

El procedimiento para la estimación de la posición del frente de avance, al interior de la muestra de roca, se presenta en el ALGORITMO 3.

\section{Algoritmo de estimación de volumen atrapado}

Conociendo la porosidad de la muestra de roca y la saturación de crudo y agua, para cada corte transversal, es posible entonces calcular el volumen de agua dopada y crudo en cualquier j-instante e i-corte transversal al interior de la muestra de roca, de la siguiente manera:

$$
\begin{gathered}
V_{\phi i}=V_{i} * \phi_{i} \\
V_{o i j}^{R}=V_{\phi i} * S_{o i j}^{R} \\
V_{w i j}^{R}=V_{\phi i} * S_{w i j}^{R}
\end{gathered}
$$

donde $\phi_{i}$ es la porosidad del i-esimo corte transversal, $V_{i}$ es el volumen escaneado del i-esimo corte transversal, y $V_{\phi i}$ es el volumen poroso efectivo en el i-esimo corte transversal. Se obtienen entonces los volúmenes de agua dopada, $V_{o i j}^{R}$, y crudo, $V_{o i j}^{R}$ atrapados al interior de la roca para cada i-corte transversal, en cada j-instante de tiempo tal y como se indica en el ALGORITMO 4.

ALGORITMO 3. Estimación de estimación de la posición del frente de avance para cualquier j-instante temporal

Entrada: Superficie de saturación de crudo vs tiempo vs corte transversal.

Salida: Posición del frente de avance para cada j-instante de tiempo.

1. para cada $\mathrm{j}$-instante temporal hacer

2. para cada $\mathrm{i}$-corte transversal hacer

3. resolver

4. fin para

$$
f_{i}=\left|\frac{d S_{o}^{R}}{d t}\right|
$$

5. establecer

$$
F A_{j}=i \mid f_{i}^{*}=\max \left(f_{i}\right) \forall i-\text { corte transversal }
$$

\section{6. fin para}

ALGORITMO 4. Estimación volúmenes de agua dopada y crudo al interior de la roca para cualquier j-instante temporal

Entrada: Superficie de concentración de crudo vs tiempo $v s$ corte transversal. Porosidad efectiva $\phi_{i}$ para cada i-corte transversal. Volumen $V_{i}$ de cada i-corte transversal.

Salida: Volúmenes de agua dopada y crudo atrapados al interior de la roca, para cada i-corte transversal y $\mathrm{j}$-instante de tiempo.

1. para cada $\mathrm{j}$-instante temporal hacer

2. para cada i-corte transversal hacer

3. resolver

$$
\begin{gathered}
V_{\phi i}=V_{i} * \phi_{i} \\
V_{o i j}^{R}=V_{\phi i} * S_{o i j}^{R} \\
V_{w i j}^{R}=V_{\phi i} * S_{w i j}^{R}
\end{gathered}
$$

\section{4. fin para}

5. resolver

$$
\begin{aligned}
& V_{o j}^{R}=\sum_{i=1}^{N} V_{o i j}^{R} \\
& V_{w j}^{R}=\sum_{i=1}^{N} V_{w i j}^{R}
\end{aligned}
$$

6. fin para 


\section{Algoritmo de estimación del factor de recobro}

Conocido el volumen de crudo atrapado al interior de la roca, en cualquier $\mathrm{j}$-instante de tiempo, es posible entonces compararlo con el volumen de crudo en la roca al inicio del experimento de desplazamiento, así:

$$
F R_{j}=\left(\frac{V_{i=0}^{R}-V_{o j}^{R}}{V_{o j=0}^{R}}\right) * 100
$$

donde $V_{o j}^{R}$ es el volumen de crudo atrapado al interior de la roca en un j-instante de tiempo y $V_{o j=0}^{R}$ es el volumen de crudo atrapado al interior de la roca en el instante inicial del experimento de desplazamiento. El procedimiento para la estimación del Factor de Recobro, para cada j-instante de tiempo se muestra en el ALGORITMO 5.

ALGORITMO 5. Estimación del Factor de Recobro para cualquier j-instante temporal

Entrada: Volúmenes de crudo atrapados al interior de la roca para cada j-instante de tiempo.

Salida: Factor de Recobro para cada j-instante de tiempo.

1. para cada $\mathrm{j}$-instante temporal hacer

2. resolver

$$
F R_{j}=\left(\frac{V_{o j=0}^{R}-V_{o j}^{R}}{V_{o j=0}^{R}}\right) * 100
$$

\section{3. fin para}

\section{ANÁLISIS DE LOS RESULTADOS DE LOS ALGORITMOS}

La estimación de la porosidad efectiva de la muestra de roca fue del 19,4\%, en promedio, usando Tomografía Computarizada, frente al $20 \%$ calculado mediante la norma API RP-40. Esto es un error relativo del 2,5\%, el cual está dentro del margen aceptable. La FIGURA 3 muestra la porosidad efectiva para cada i-corte transversal, calculada a partir del ALGORITMO 1, donde se observa el comportamiento heterogéneo de la muestra de roca, con una menor porosidad al frente de esta.

La FIGURA 4 y la FIGURA 5 presentan la saturación de crudo y agua dopada, respectivamente, al interior de la roca, calculada a partir del ALGORITMO 2. Se observa que para la hora 0,25 (15 min) el frente de avance ha alcanzado el $45 \%$ de la muestra de roca (aproximadamente), a la hora 0,35 (21 min) el frente de avanzado ha llegado al $80 \%$ de la muestra de roca, y hay rompimiento a partir de la hora 0,4 (24 min). Es decir, durante los primeros 24 min se realiza el mayor desplazamiento de crudo al interior de la roca. Después de este momento la variación de la saturación de crudo y agua al interior de la roca es más lenta. Es de notarse, además, que la saturación de crudo y agua es menor en el frente de la muestra de roca debido a la menor porosidad de esta sección.

El frente de avance se presenta en la FIGURA6, calculada a partir del ALGORITMO 3, donde el mayor valor de la derivada (puntos más brillantes) indican la posición del frente de avance para un instante de tiempo. Nótese que en la hora 0,25 (15 min) se presentan dos frentes de avance claramente diferenciados. Esto es debido a que la estructura porosa ha favorecido la creación de un segundo canal preferencial de desplazamiento. En la hora 0,18 (10 min) y 0,35 (21 min) se observa un frente de avance principal con una serie de frentes de avance secundarios más pequeños.

En la FIGURA 6 solo se observan 3 posiciones del frente de avance debido a que la velocidad del desplazamiento del agua dopada (desplazando el crudo al interior de la roca) provocó que hubiera rompimiento antes de la cuarta captura CT.

Los volúmenes de agua y crudo atrapados al interior de la roca se presentan en la FIGURA 7, calculada a partir del ALGORITMO 4. El volumen total de crudo desplazado, medido experimentalmente, fue de 8200 $\mathrm{mm}^{3}$ frente a los $8150 \mathrm{~mm}^{3}$ estimados mediante el uso de imágenes CT. Esto es un error relativo menor al 1\%. Por último, el factor de recobro estimado se presenta en la FIGURA 8. El factor de recobro estimado experimentalmente fue del $65 \%$ frente a un $62 \%$ estimado usando imágenes CT, lo que representa un error relativo del 5\%. De nuevo, es un error aceptable.

En la FIGURA 7 se observa un ligero incremento del volumen de crudo y en la FIGURA 8, calculada a partir del ALGORITMO 5, se observa una ligera caída del factor de recobro al final del desplazamiento. Esto es debido al ruido experimental de la Tomografía Computarizada y errores numéricos acumulados de la estimación de la porosidad, saturación y volumen atrapado. Este error numérico es aditivo y se puede reducir con un desarrollo experimental más preciso, sin embargo es inevitable que suceda. 


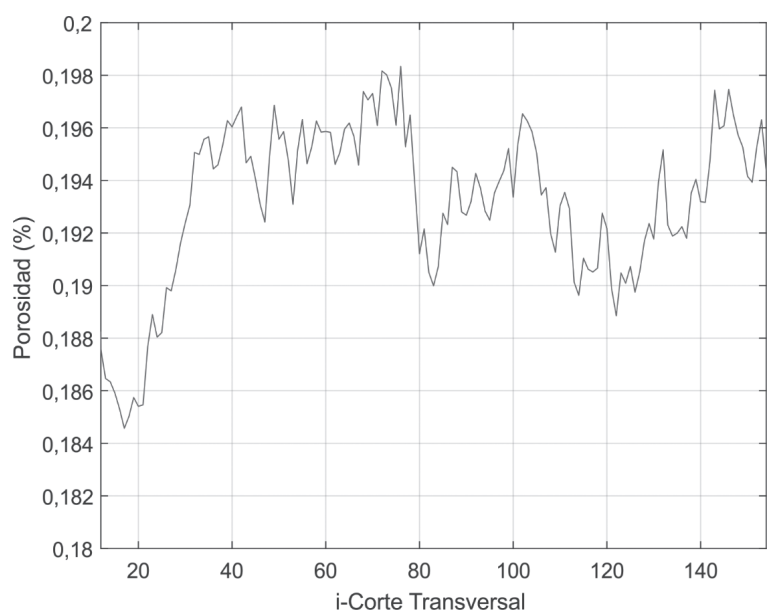

FIGURA 3. Porosidad $\phi$ estimada para cada i-corte transversal.

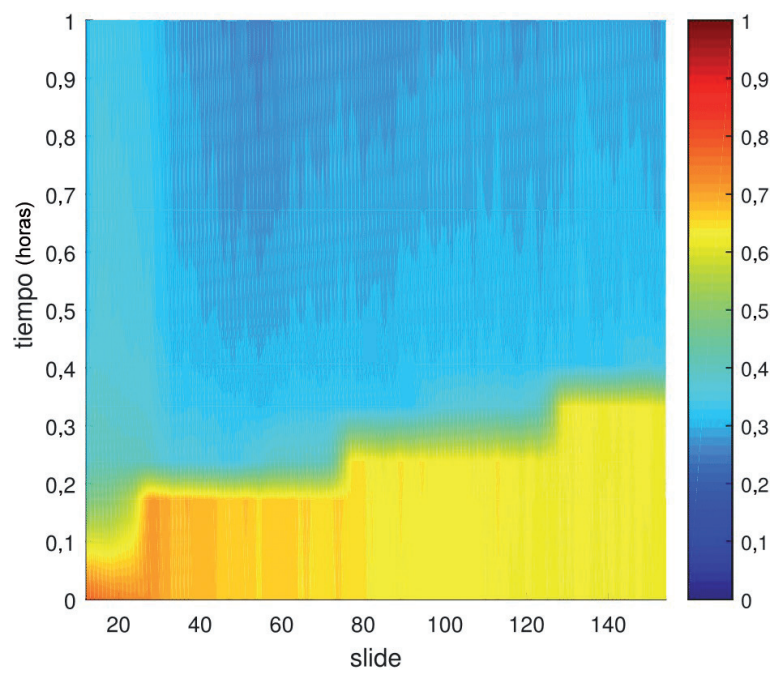

FIGURA 4. Saturación de crudo al interior de la roca para cada i-corte transversal y j-instante de tiempo.

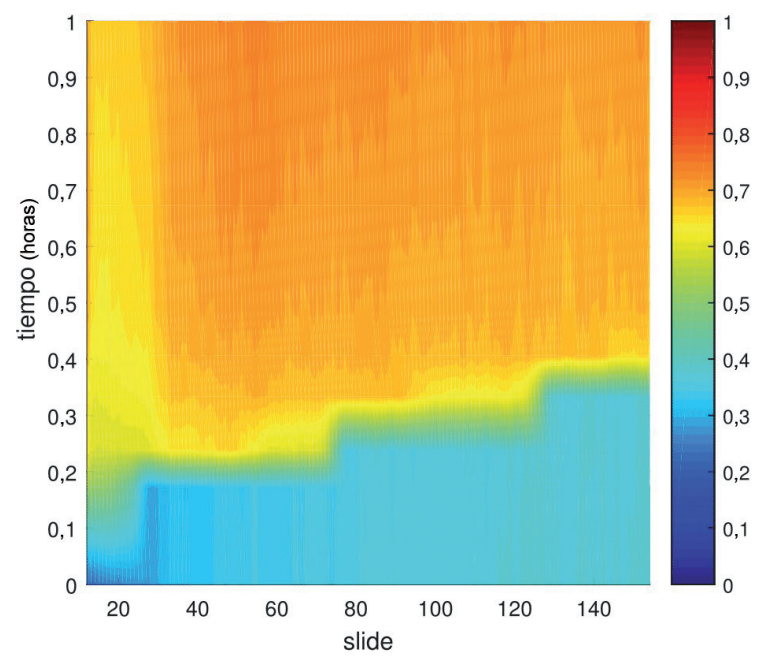

FIGURA 5. Saturación de agua dopada al interior de la roca para cada i-corte transversal y j-instante de tiempo.

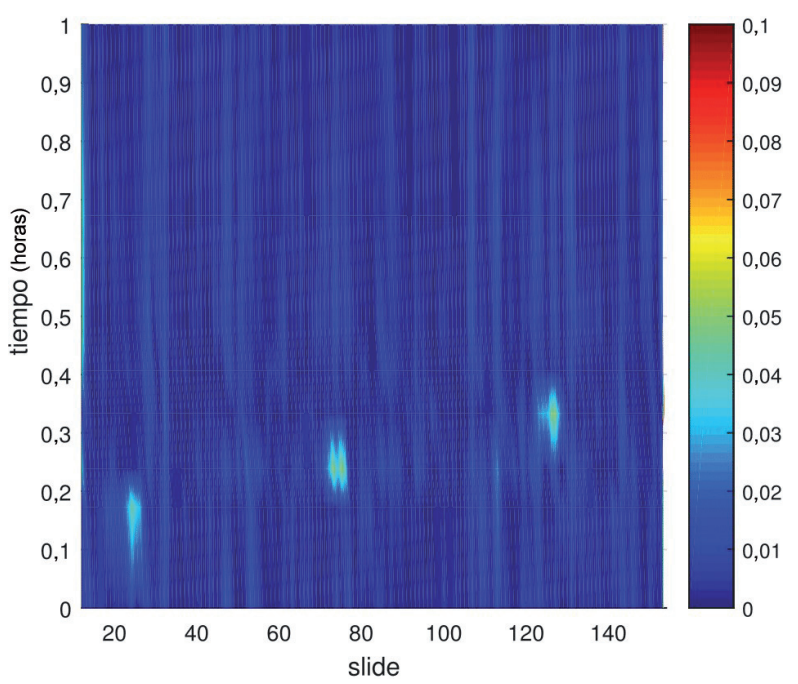

FIGURA 6. Posición del Frente de Avance para cada i-corte transversal y j-instante de tiempo.

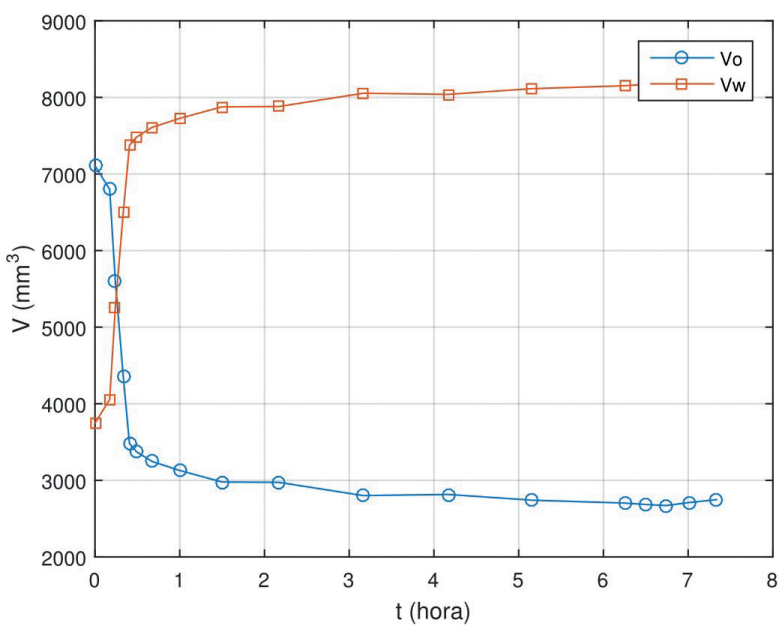

FIGURA 7. Volúmenes de crudo y agua dopada atrapados al interior de la muestra de roca para cada j-instante de tiempo.

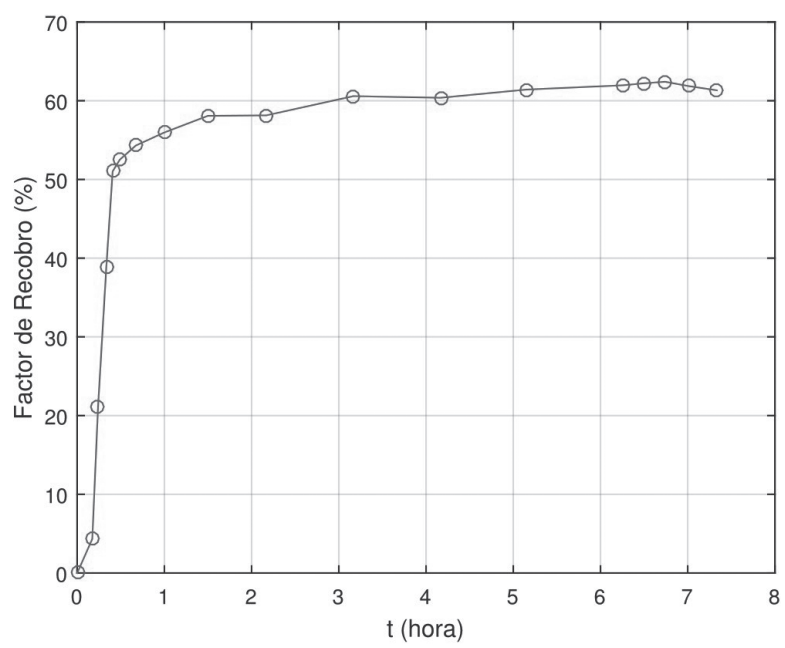

FIGURA 8. Factor de Recobro para cada j-instante de tiempo. 


\section{CONCLUSIONES}

El uso de Tomografía Computarizada para evaluar el desplazamiento de dos fases líquidas, al interior de una muestra de roca, requiere de un mayor esfuerzo experimental, si se le compara con el estándar API PR-40. Es necesario tener información de referencia de la atenuación provocada por cada una de las dos fases líquidas además del medio poroso saturado al $100 \%$ por cada fase. Cada muestreo tomado durante el desplazamiento de las dos fases líquidas genera un cubo de datos con cerca de 150 cortes transversales por muestreo. El volumen de datos generados por experimento de desplazamiento es inmenso.

Sin embargo, la Tomografía Computarizada permite evaluar en cada instante de tiempo, y en cada corte transversal, la saturación, concentración y volumen atrapado en cada sección de la muestra de roca. Conociendo la variación en el tiempo de la saturación de crudo, o agua, en una sección en particular de la muestra de roca es posible detectar problemas en la implementación de técnicas de recobro mejorado. Problemas como zonas de menor porosidad, canales de flujo preferentes, taponamiento de la muestra de roca, entre otros. En contraste, las técnicas clásicas solo permiten estimar el comportamiento del desplazamiento de manera general, comparando el flujo de entrada $v s$ el flujo de salida.

Aunque los experimentos aquí mencionados solo incluyen el desplazamiento de crudo usando agua dopada, es posible realizar el mismo trabajo experimental usando agua diluida con polímeros y/o surfactantes, y contrastar los resultados con el uso de agua dopada. Esta comparación es, como conclusión, la efectividad de un agente de recobro mejorado vs un patrón de inyección de agua. Esta comparación es posible, además, gracias a que el uso de Tomografía Computarizada no compromete la integridad física de la roca, pudiendo ser reutilizada en nuevos experimentos de desplazamiento.

\section{AGRADECIMIENTOS}

Los autores quieren agradecer a Colciencias y a Ecopetrol S.A. por su apoyo y participación en la convocatoria de Colciencias 531-2011 Técnicas avanzadas de imágenes en medios porosos para la caracterización no intrusiva de roca e incremento del factor de recobro en campos de crudo pesado, y campos maduros de crudos convencionales, en la cual se enmarca el presente trabajo de investigación.

\section{NOMENCLATURA}

Coeficientes de atenuación de las diferentes

$\mu(\eta)$ secciones de un material heterogéneo, en la dirección $\eta$ de propagación del haz de rayos-X

Proyección de la energía del haz de rayos $\mathrm{X}$

$p(s)$ sobre la energía captada por los sensores, al recorrer una distancia $s$

Coeficientes de atenuación de las diferentes

$\mu(\varepsilon, \eta)$ secciones de un material heterogéneo, en el sistema de rotación $(\varepsilon, \eta)$, de propagación del haz de rayos- $X$

Proyección de la energía del haz de rayos X

$p_{\gamma}(s)$ sobre la energía captada por los sensores, al recorrer una distancia $s$, para un ángulo $\gamma$

A Matriz que modela el sistema (transformación al espacio Radon, $\mathbf{R}$ )

R Espacio Radón

Contiene todos los valores de grises de la rejilla (imagen en el espacio cartesiano (x, y))

l. Número de cortes transversales (imágenes $\mathrm{CT}$ ) generados en un muestreo CT

$m \quad$ Número de filas en cada corte transversal

$n \quad$ Número de columnas en cada corte transversal Índice del corte transversal (imagen CT) dentro del cubo de datos

$j$ Índice del instante temporal en el que fue escaneada la muestra de roca

Porosidad (\%) promedio del i-ésimo corte

$\phi_{i}$ transversal

$C T_{0=1}^{R} \quad$ Cubo de datos de imágenes $\mathrm{CT}$ de una muestra de roca totalmente saturada de crudo

$C T^{R} \quad$ Cubo de datos de imágenes $\mathrm{CT}$ de una muestra

$C T_{w=1}$ de roca totalmente saturada de agua dopada

$C T_{o}$ Valor de atenuación del crudo

$C T_{w}$ Valor de atenuación del agua dopada

Valor CT promedio del i-ésimo corte transversal

$C T_{o i}^{R}$ del cubo de datos de imágenes CT de una muestra de roca totalmente saturada de crudo

Valor CT promedio del i-ésimo corte $C T_{w i}^{R}$ transversal del cubo de datos de imágenes CT de una muestra de roca totalmente saturada de agua dopada

Valor CT promedio del i-ésimo corte transversal del cubo de datos de imágenes CT

$C T_{i j}^{R} \quad$ de una muestra de roca escaneada en el j-ésimo instante del desplazamiento de crudo usando agua dopada 
Saturación promedio de agua dopada al interior de la roca en el i-ésimo corte transversal del $S_{w i j}^{R} \quad$ cubo de datos de imágenes CT, en el j-ésimo instante del desplazamiento de crudo usando agua dopada

Saturación promedio de crudo al interior de la roca en el i-ésimo corte transversal del cubo de

$S_{o i j}^{R} \quad$ datos de imágenes CT, en el j-ésimo instante del desplazamiento de crudo usando agua dopada

Posición del frente de avance en el j-ésimo

$F A_{j} \quad$ instante del desplazamiento de crudo usando agua dopada

$V_{i} \quad$ Volumen escaneado del i-ésimo corte transversal del cubo de datos de imágenes $\mathrm{CT}$

$V$ Volumen poroso efectivo en el i-ésimo corte transversal del cubo de datos de imágenes CT

Volumen de crudo atrapado en el i-ésimo corte $V_{o i j}^{R}$ transversal del cubo de datos de imágenes CT, en el j-ésimo instante del desplazamiento de crudo usando agua dopada

Volumen de agua dopada atrapado en el i-ésimo corte transversal del cubo de datos de imágenes

$V_{w i j}^{R} \quad \mathrm{CT}$, en el j-ésimo instante del desplazamiento de crudo usando agua dopada

Factor de recobro alcanzado en el j-ésimo

$F R_{j}$ instante del desplazamiento de crudo usando agua dopada

\section{REFERENCIAS}

ANH. 2016. Sistema Integrado de Reservas. Agencia Nacional de Hidrocarburos. Consultado el 01 de enero de 2016. http://www.anh.gov.co/Operaciones-Regaliasy-Participaciones/Paginas/Sistema-Integrado-deReservas.aspx

API RP-40. Recommended Practices for Core Analysis. Section 1- Planning a Coring Program. American Petroleum Institute. 1998.

Bansal, A., and Islam, M.R. 1991. State-of-the-art review of nondestructive testing with computerassisted tomography. International Arctic Technology Conference. Society of Petroleum Engineers. Anchorage, Estados Unidos, pp. 523-534.

Blau, S., Robertson, S., and Johnstone, M. 2008. Disaster victim identification: new applications for postmortem computed tomography. Journal of Forensic Sciences, 53(4): 956-961.
Borkan, G.A., Hults, D.E., Gerzof, S.G., Robbins, A.H., and Silbert, C.K. 1983. Age changes in body composition revealed by computed tomography. Journal of Gerontology, 38(6): 673-677.

Buzug, T.M. 2008. Computed Tomography: From photon statistics to modern cone-beam CT. Springer. Leipzig, 522p.

Castro, R., Maya, G., Mercado, D., Trujillo, M., Soto, C., Pérez, H., Lobo, A., Ordóñez, A., and Sandoval, J.E. 2010. Enhanced Oil Recovery (EOR) Status - Colombia. SPE Latin American and Caribbean Petroleum Engineering Conference, Lima, Peru. SPE-139199-MS.

Cozzini, C., Olesinski, S., and Harding, G. 2012. Modeling scattering for security applications: A multiple beam X-Ray Diffraction Imaging system. IEEE Nuclear Science Symposium and Medical Imaging Conference, Anaheim, Estados Unidos, pp. 74-77.

Fajardo, R.J., and Müller, R. 2001. Three-dimensional analysis of nonhuman primate trabecular architecture using micro-computed tomography. American Journal of Physical Anthropology, 115(4): 327-336.

Hackley, P.C., Urbani, F., Karlsen, A.W., and Garrity, C.P. 2006. Mapa geológico de Venezuela a Escala 1:750.000. USGS.

Hunt, P., Engler, P., and Bajsarowicz, C. 1988. Computed tomography as a core analysis tool: Applications, instrument evaluation, and image improvement techniques. Journal of Petroleum Technology, 40(9): 1203-1210.

Kantzas, A. 1990. Investigation of physical properties of porous rocks and fluid flow phenomena in porous media using computer assisted tomography. In Situ, 14(1): 77-132.

Kellinghaus, M., Schulz, R., Vieth, V., Schmidt, S.,Pfeiffer, H., and Schmeling, A. 2010. Enhanced possibilities to make statements on the ossification status of the medial clavicular epiphysis using an amplified staging scheme in evaluating thin-slice CT scans. International Journal of Legal Medicine, 124(4): 321-325.

Meyer, R.F., Attanasi, E.D., and Freeman, P.A., 2007. Heavy oil and natural bitumen resources in geological basins of the world: U.S. Geological Survey Open-File Report 2007-1084. 
OPEC. 2016. OPEC - Oil data: upstream. Consultado el 1 de diciembre de 2016. http://asb.opec.org/index.php/ interactive-charts/oil-data-upstream

Owen, N., Inderwildi, O., and King, D. 2010. The status of conventional world oil reserves-Hype or cause for concern?. Energy Policy, 38(8): 4743-4749.

Pani, S., Cook, E., Horrocks, J., George, L., Hardwick, S., and Speller, R. 2009. Modeling an energy-dispersive $\mathrm{X}$-ray diffraction system for drug detection. IEEE Transactions on Nuclear Science, 56(3): 1238-1241.

SGC. 2015. Mapa Geológico de Colombia. Servicio Geológico Colombiano. Consultado el 01 de diciembre de 2016. http://geoportal.sgc.gov.co/geoportalsgc/ catalog/main/home.page

Shameem, S., and Khamees, A. 2004. Dual-Energy CTScanning Applications in Rock Characterization. SPE Annual Technical Conference and Exhibition. Houston, Estados Unidos, pp. 26-29.

Shannon, C. E. 1949. Communication in the presence of noise. Proceedings of the IRE, 37(1): 10-21.

Smith, F. 1999. Industrial Applications of X-Ray Diffraction. CRC Press. Nueva York, 1006p.

Speight, J.G. 1997. Petroleum chemistry and refining. CRC Press. Nueva York, 350p.

Strecker, H. 1998. Automatic detection of explosives in airline baggage using elastic X-ray scatter. MedicaMundi, 42(2): 30-33.

Tafforeau, P., Boistel, R., Boller, E., Bravin, A., Brunet, M., Chaimanee, Y., and Kay, R.F. 2006. Applications of X-ray synchrotron microtomography for nondestructive 3D studies of paleontological specimens. Applied Physics A, 83(2): 195-202.

Thali, M.J., Taubenreuther, U., Karolczak, M., Braun, M., Brueschweiler, W., Kalender, W., and Dirnhofer, R. 2003a. Forensic microradiology: Micro-Computed Tomography (Micro-CT) and analysis of patterned injuries inside of bone. Journal of Forensic Sciences, 48(6): 1336-1342.

Thali, M. J., Yen, K., Schweitzer, W., Vock, P., Ozdoba, C., and Dirnhofer, R. 2003b. Into the decomposed bodyforensic digital autopsy using Multislice-Computed
Tomography. Forensic Science International, 134(2): 109-114.

Uda, M., Demortier, G., and Nakai, I. 2005. X-rays for Archaeology. Amsterdam, Dordrecht, The Netherlands. Springer, 301p.

Wellington, S.L., and Vinegar, H.J. 1987. X-ray computerized tomography. Society of Petroleum Engineers, 39(8): 885-898.

Wheeler, J.O., Hoffman, P.F., Card, K.D., Davidson, A., Sanford, B.V., Okulitch, A.V., and Roest, W.R. 1996. Geological Map of Canada, Map D1860A, Geological Survey of Cannada, Ottawa, Ontario.

Withjack, E.M. 1988. Computed tomography for rockproperty determination and fluid-flow visualization. SPE Formation Evaluation, 3(4): 696-704.

Trabajo recibido: abril 07 de 2017

Trabajo aceptado: agosto 31 de 2017 